\title{
Análise da obra: Viana, Renata Andrea Pietro Pereira. Sepse: um problema de saúde pública - A atuação e colaboração da Enfermagem na rápida identificação e tratamento da doença (ISBN 978-85-68720-05-9)
}

Analysis of the work: Viana, Renata Andrea Pietro Pereira. Sepsis: a public health problem - Nursing's performance and collaboration in the rapid identification and treatment of the disease (ISBN 978-85-68720-05-9)

Análisis de la obra: Viana, Renata Andrea Pietro Pereira. Sepsis: Sepse: un problema de salud pública - La actuación y colaboración de la Enfermería en la rápida identificación y tratamiento de la enfermedad (ISBN 978-85-68720-05-9)

Marcelo Ricardo Rosa ${ }^{1 *}$, Cely de Oliveira ${ }^{1}$, Walace Fernando Rocha de Souza ${ }^{1}$, Ridenia Cavalcante Pessoa ${ }^{1}$, Eneida Tramontina Valente Cerqueira ${ }^{1}$, João Gabriel de Souza Almeida1', Andréa de Oliveira Carvalho Aragão ${ }^{1}$

\section{RESUMO}

Objetivo: Despertar nos profissionais e acadêmicos de Enfermagem e outras áreas das ciências da saúde a conscientização e importância na leitura da obra para processo de identificação, cuidados e tratamento no atendimento ao paciente séptico. Métodos: Análise descritiva. Discussão: A análise da obra Sepse: um problema de saúde pública - A atuação e colaboração da Enfermagem na rápida identificação e tratamento da doença demonstra um aumento significativo de casos de sepses em unidades de saúde e pouco conhecimento das equipes sobre 0 assunto. Considerações finais: Faz-se necessário que os profissionais da enfermagem e de outras áreas das ciências da saúde, estejam em constante desenvolvimento profissional, aprimorando seus conhecimentos, principalmente em agravos a saúde humana. No caso da sepse, sendo hoje considerada um grave problema de saúde pública, a obra apresentada vem proporcionando aos seus leitores grandes contribuições para o planejamento da assistência e para a tomada de decisão clínica baseada em evidências.

Palavras-chave: Sepse, Identificação, Enfermagem.

\section{ABSTRACT}

Objective: To awaken in the professionals and academics of Nursing and other areas of the health sciences the awareness and importance in the reading of the work for the process of identification, care and treatment in the care of the septic patient. Methods: Descriptive analysis. Discussion: The analysis of the work Sepse: a public health problem - Nursing's performance and collaboration in the rapid identification and treatment of the disease has been showing a significant increase in cases of sepsis in health units and little knowledge of the teams on the subject. Final considerations: It is necessary that nursing professionals and other areas of the health sciences, are in constant professional development, improving their knowledge, mainly in human health problems. In the case of sepsis, which is now considered a serious public health problem, the work presented has provided its readers with great contributions to care planning and evidence-based clinical decision-making.

Key words: Sepsis, Identification, Nursing.

${ }^{1}$ Centro Universitário São Judas Tadeu, Santos, SP. * E-mail: $\underline{\text { mrosa.ead@gmail.com }}$

SUBMETIDO EM: 5/2018

ACEITO EM: 6/2018

PUBLICADO EM: 12/2018 


\section{RESUMEN}

Objetivo: Despertar en los profesionales y académicos de Enfermería y otras áreas de las ciencias de la salud la concientización e importancia en la lectura de la obra para el proceso de identificación, cuidados y tratamiento en la atención al paciente séptico. Métodos: Análisis descriptivo. Discusión: La actuación y colaboración de la Enfermería en la rápida identificación y tratamiento de la enfermedad viene demostrando un aumento significativo de casos de sepsis en unidades de salud y poco conocimiento de los equipos sobre el tema. Consideraciones finales: Se hace necesario que los profesionales de la enfermería y de otras áreas de las ciencias de la salud, estén en constante desarrollo profesional, mejorando sus conocimientos, principalmente en agravios a la salud humana. En el caso de la sepsis, siendo hoy considerada un grave problema de salud pública, la obra presentada viene proporcionando a sus lectores grandes contribuciones para la planificación de la asistencia y para la toma de decisión clínica basada en evidencias.

Palabras clave: Sepse, Identificación, Enfermería.

A autoria da obra ficou a cargo de Renata Andréa Pietro Pereira Viana, doutora em Ciências da Saúde, Conselheira titular do COREN-SP; Flavia Ribeiro Machado, doutora em Doenças Infecciosas e Parasitárias pela Universidade de São Paulo, coordenadora geral do ILAS; Juliana Lubarino Amorim de Souza, Enfermeira intensivista graduada pela Universidade Federal de São Paulo, enfermeira coordenadora do ILAS. Na compilação do assunto em 65 páginas repletas de informações cuidadosamente selecionadas a fim de explanar a necessidade do conhecimento científico de um assunto importante e de valor considerável para um prognóstico positivo do assistido. Na obra Sepse: um problema de saúde pública - A atuação e colaboração da Enfermagem na rápida identificação e tratamento da doença, o Conselho Regional de Enfermagem de São Paulo (COREN), e o Instituto Latino Americano para Estudos da SEPSE (ILAS), se associam para elaborar um manual com onze capítulos que aborda os principais aspectos da sepse e os cuidados que pode ser realizado para minimizar a morbidade. Essa segunda publicação apresentada em 2017, denota o papel essencial que a enfermagem exerce no cuidado e prevenção da sepse

De maneira clara e concisa, a autora perpassa por todos os tópicos da fisiologia e fisiopatologia atrelada à sepse: definições e conceitos, dados epidemiológicos, importância da identificação rápida da sepse. Posteriormente, descreve o impacto da sepse em todos os sistemas orgânicos, a instituição do tratamento e seus aspectos, a elaboração dos protocolos assistenciais e a importância da equipe de enfermagem no reconhecimento e tratamento da sepse. Finalizando, temos os diagnósticos e intervenções de enfermagem que norteiam as condutas do enfermeiro auxiliando, portanto, de maneira eficaz a gestão do cuidado voltado ao quadro séptico.

Em primeiro momento, vemos a descrição das características fisiopatológicas da sepse que, de acordo com a autora, a diferenciação entre Síndrome da Resposta Inflamatória Sistêmica (SRIS) e sepse, por vezes, é difícil porque nem sempre é clara a presença de foco infeccioso. Esse diagnóstico diferencial torna-se um desafio, por exemplo, em pacientes com SRIS secundária a politrauma ou cirurgia de grande porte. Caso esses pacientes passem a ter um foco infeccioso, seu diagnóstico correto é dificultado pela presença dos sinais de resposta inflamatória em resposta à agressão anterior. Por outro lado, pacientes imunossuprimidos ou com idade avançada podem manifestar disfunção orgânica e mesmo choque séptico, sem a presença dos sinais clássicos de SRIS (SEYMOUR, et al, 2016).

Viana (2017) discorrendo sobre o impacto da sepse em todos os sistemas orgânicos, informa-nos que a sepse se caracteriza pela presença de sinais de disfunção orgânica, com manifestações clínicas decorrentes dos órgãos em disfunção. O choque séptico é o de mais pronto diagnóstico, pois a hipotensão é facilmente perceptível. Entretanto, o diagnóstico nessa fase pode ser considerado tardio. Todos os esforços devem ser feitos no sentido de diagnosticar a sepse em seus estágios iniciais, quando a intervenção tem maiores possibilidades de evitar o óbito. Neste contexto, o papel do enfermeiro é primordial, já que este está presente, de maneira constante, à beira do leito do paciente. A redução da oferta de oxigênio e as alterações celulares levam à disfunção orgânica. As principais disfunções são: neurológica, respiratória, cardiovascular, gastrintestinal, renal, hematológica e endocrinológica. 
No capítulo subsequente, a formulação das ideias frente ao problema representado, a sepse, pela elevada incidência, altos custos e mortalidade, o principal desafio dos prestadores de serviço à saúde é implementar, de forma institucionalmente gerenciada, programas que levem à beira do leito as melhores evidências científicas disponíveis, visando garantir a melhor prática assistencial. Na sepse, as diretrizes para tratamento são bem estabelecidas, o que teoricamente dá sustentação à implementação de processos adequados de assistência.

A equipe de Enfermagem tem um papel relevante no diagnóstico e tratamento do paciente séptico, devido ao fato de permanecer, a maior parte do tempo, à beira do leito, identificando e atuando frente às necessidades humanas básicas afetadas e contribuindo com a equipe multiprofissional na instituição de tratamentos e cuidados pertinentes, precocemente, o que pode contribuir para o aumento da sobrevida. Nesse âmbito, os capítulos 10 e 11 trata amplamente dos conhecimentos e do papel da enfermagem para que as necessidades humanas dos pacientes sépticos sejam atendidas.

A Society of Critical Care Medicine (SCCM) e a European Society of Critical Care Medicine (ESICM) chegaram a um consenso e novas definições de sepse foram publicadas, conhecidas como Sepsis 3, definida pela "presença de disfunção orgânica ameaçadora à vida secundária à resposta desregulada do organismo à infecção" (SINGER et al, 2016)

Shankar-Hari (2016) afirma que para avaliação do diagnóstico clínico de disfunção orgânica, atualmente são observados uma variação de dois ou mais pontos no escore Sequential Organ Failure Assessment (SOFA). Os critérios da síndrome da resposta inflamatória sistêmica (SRIS) não são mais usados para a definição. Os casos de sepse passam a ser considerados como doença grave, assim, não se usa mais a expressão "sepse grave". Choque séptico é definido como "um subgrupo dos pacientes com sepse que apresentam acentuadas anormalidades circulatórias, celulares e metabólicas e associadas com maior risco de morte do que a sepse isoladamente". Os critérios diagnósticos de choque séptico são a "necessidade de vasopressor para manter uma pressão arterial média acima de $65 \mathrm{mmHg}$ após a infusão adequada de fluidos, associada a nível sérico de lactato acima de $2 \mathrm{mmol} / \mathrm{L}$ ".

Um dos principais fatores indiretamente relacionados com a sepse é o tempo de permanência do paciente em uma UTI. Quanto mais tempo permanecer, maior é a chance de desenvolver uma infecção (COSTA et al, 2008) o que reforça o que é indicado no capítulo 11 referente ao diagnóstico e intervenção da enfermagem de acordo com Viana, 2017: "os profissionais de Enfermagem são os que permanecem a maior parte do tempo à beira do leito, por isso devem estar aptos a identificar os sinais e sintomas da sepse e planejar a assistência de Enfermagem, de acordo com as necessidades de cada paciente. Nesse cenário, a atualização e a competência do enfermeiro tornam-se obrigatórias quando a finalidade é garantir um cuidado de Enfermagem de qualidade".

Renata Andrea Pietro Pereira Viana é Graduada em Enfermagem e Obstetrícia pela Faculdade de Medicina de Marília - FAMEMA (1998), com especialização em Nefrologia pela Universidade Federal de São Paulo - UNIFESP (2000), Epidemiologia Hospitalar pela UNIFESP (2005), Administração Hospitalar pela UNAERP (2005) e Educação em Saúde pela UNIFESP (2006), Fundadora e membro efetivo da Associação Brasileira de Enfermagem em Terapia Intensiva (ABENTI), com Proficiência em Terapia Intensiva pela ABENTI (2013). Ex-presidente do Departamento de Enfermagem da Associação de Medicina Intensiva Brasileira - AMIB nos biênios 2009-2010, 2010-2011. Mestrado em Educação e Saúde pela UNIFESP (2008). Doutorado em Ciências da Saúde pela UNIFESP (2013). Atualmente é Diretora do Núcleo de Terapia Intensiva do Hospital do Servidor Público Estadual de São Paulo - Gerência de Enfermagem. Pesquisadora do Grupo de Estudos e Pesquisa em Administração em Saúde e Gerenciamento de Serviços de Enfermagem (GEPAG/UNIFESP). Conselheira Regional Titular no COREN-SP triênio 2015-2017, responsável pelo COREN-SP Educação. Pesquisadora e orientadora do Programa de Mestrado Profissional da Pós-Graduação em Ciências da Saúde do IAMSPE-SP desde o ano de 2013. Membro do Corpo Editorial da Revista brasileira de Terapia Intensiva desde 2009, revisora do International Scholars Journals desde 2013 e da Revista da Escola de Enfermagem da USP desde 2015. Presidente do Conselho Regional de Enfermagem de São Paulo (COREN-SP), gestão 2018/2020. Dentre suas produções, destacam-se: Enfermagem em Terapia Intensiva: 
Práticas Integrativas; Sepse para Enfermeiros: As horas de ouro - Identificando e cuidando do paciente séptico; Enfermagem em Terapia Intensiva Desmistificada: Um guia de aprendizado; Enfermagem em Terapia Intensiva: Práticas e Vivências; Sepse para Enfermeiros - As horas de ouro: Identificando e cuidando do paciente séptico.

Esta resenha descritiva teve por objetivo analisar e descrever a obra "Sepse: um problema de saúde pública - A atuação e colaboração da Enfermagem na rápida identificação e tratamento da doença", pelos autores Marcelo Ricardo Rosa, enfermeiro, mestre, docente no curso de enfermagem no Centro Universitário São Judas Tadeu - campus Unimonte e Walace Fernando Rocha de Souza, biomédico, mestrando em Hemoterapia e acadêmico do curso de Enfermagem no Centro Universitário São Judas Tadeu - campus Unimonte

\section{REFERÊNCIAS}

1. Costa JB, Marcon SS, Mombelli MA. Caracterização das internações em uma unidade de terapia intensiva- UTI adulto de um hospital escola. Ciências Cuidado Saúde. 2008;7:1-4.

2. Seymour CW, Liu VX, Iwashyna TJ, Brunkhorst FM, Rea TD, Scherag A, et al. Assessment of Clinical Criteria for Sepsis: For the Third International Consensus Definitions for Sepsis and Septic Shock (Sepsis-3). JAMA. 2016;315(8):762-74. Erratum in: JAMA. 2016;315(20):2237.

3. Shankar-Hari M, Phillips GS, Levy ML, Seymour CW, Liu VX, Deutschaman CS, et al. Sepsis definitions task force developing a new definition and assessing new clinical criteria for septic shock: for the third international consensus definitions for sepsis and septic shock (sepsis-3). JAMA. 2016;315(8):775-87.

4. Singer M, Deutschman CS, Seymour CW, Shankar-Hari M, Annane D, Bauer M, et al. The third international consensus definitions for sepsis shock (sepsis-3). JAMA. 2016;315(8):801-10. doi:10.1001/jama.2016.0287.

5. Viana RAPP, Machado FR, Souza JLA. Sepse, um problema de saúde pública: a atuação e colaboração da enfermagem na rápida identificação e tratamento da doença. $2^{\underline{a}}$ ed. São Paulo: COREN-SP; 2017. ISBN 978-85-68720-05-9. 